\title{
Arterial Stiffness and Central Hemodynamics are Associated with Low Diurnal Urinary Sodium Excretion
}

This article was published in the following Dove Press journal: Diabetes, Metabolic Syndrome and Obesity: Targets and Therapy

\author{
Rosaria Del Giorno (iD) ${ }^{1,2, *}$ \\ Christos Ceresa ${ }^{\mathrm{l}, *}$ \\ Sofia Gabutti ${ }^{1}$ \\ Chiara Troiani' \\ Luca Gabutti ${ }^{1,2}$ \\ 'Department of Internal Medicine, \\ Clinical Research Unit, Regional Hospital \\ of Bellinzona and Valli, Ente Ospedaliero \\ Cantonale, Bellinzona, Switzerland; \\ ${ }^{2}$ Institute of Biomedicine, University of \\ Southern Switzerland, Lugano, \\ Switzerland \\ *These authors contributed equally to \\ this work
}

Correspondence: Rosaria Del Giorno; Luca Gabutti

Department of Internal Medicine, Clinical Research Unit, Regional Hospital of Bellinzona and Valli, Ente Ospedaliero Cantonale, Bellinzona 6500, Switzerland; Institute of Biomedicine, University of Southern Switzerland, Lugano, Switzerland

Tel +4I 9I 8II $9408 ;+4$ I 9| 8II 8464 Email rosaria.delgiorno@eoc.ch; luca.gabutti@eoc.ch
Background: Excessive salt intake is an important determinant of cardiovascular (CV) health, impacting arterial stiffness and central blood pressure. However, sodium exhibits several patterns of excretion in urine during day- and night-time, which could differently affect CV risk. Here, we sought to explore the relationship between the day:night urinary sodium excretion ratio and arterial stiffness and central hemodynamics in the general population.

Methods: Cross-sectional analysis in 1062 subjects. Arterial stiffness (pulse-wave velocity, PWV), central blood pressure (central systolic blood pressure, cSBP; central diastolic blood pressure, cDBP), and other hemodynamic parameters were noninvasively assessed. Day- and night-time urinary sodium were separately detected. Analyses were performed according to the day:night urinary sodium excretion ratio tertiles (T1-T3).

Results: Low day-time excretors (T1) showed significantly higher values of arterial stiffness when compared with high day-time excretors (T3) (cf-PWV $7.6 \pm 1.9 \mathrm{vs} 6.9 \pm 1.5 \mathrm{~m} / \mathrm{sec} ; \mathrm{p} \leq$ 0.001), and higher central BP parameters (cSBP: $111.6 \pm 12.1$ vs $109.0 \pm 11.1 \mathrm{mmHg}, \mathrm{p} \leq$ 0.001 ; cDBP, $76.9 \pm 9.2$ vs $75.1 \pm 9.3 \mathrm{mmHg}, \mathrm{p} \leq 0.001)$. In multivariate linear-regression models $(\beta, C I)$, the day:night ratio of sodium excretion was significantly associated with arterial stiffness (cf-PWV $-0.386,-0.559,-0.213, \mathrm{p} \leq 0.001$ ) and with central hemodynamic parameters (cSBP $-1.655,-2.800,-0.510 ; \mathrm{p} \leq 0.001 ; \mathrm{cDBP}-1.319,-2.218,-0.420, \mathrm{p} \leq$ 0.001). Associations persisted after controlling for multiple confounding factors. In logisticregression models, the risk of increased arterial stiffness was significantly reduced as the day: night ratio of urinary sodium excretion increased (OR $0.40,95 \%$ CI $0.25-0.65, \mathrm{p} \leq 0.001$ ). Conclusion: The individual, intra-daily pattern of urinary sodium excretion, characterised by low daytime excretion, is associated with increased arterial stiffness and central blood pressure. Further studies are advocated to clarify the clinical utility of assessing the daily pattern of sodium excretion.

Keywords: night-time urinary-sodium excretion, day-time urinary-sodium excretion, arterial stiffness, pulse-wave velocity, central hemodynamics, central blood pressure

\section{Background}

Salt intake is one of the most important environmental determinants of cardiovascular health. ${ }^{1}$ Previous robust evidence highlighted the role of excessive sodium intake in promoting structural and functional pressure-independent vascular damage through the remodelling of central and peripheral arterial walls. ${ }^{2-4}$

It was postulated that high salt intake could promote oxidative stress on arterial walls, resulting in wall hypertrophy, endothelial dysfunction, and arterial-vessel 
contractility impairment, lastly increasing arterial stiffness. ${ }^{5,6}$ As a consequence, increased arterial stiffness could produce an increasing amplitude of forward and reflected waves, and an anticipated comeback to the proximal aorta of the reflected wave, as well as a central systolic blood pressure elevation (cSBP). ${ }^{7}$

Central blood pressure (cBP) is a major element able to predict target organ (such as brain, heart, and kidney) damage, and it is therefore conceivable that it could be more predictive of CVD than peripheral blood pressure (BP). A cumulative amount of evidence suggests the pivotal role of cBP in target organ damage and, more recently, metaregression analysis has revealed the higher power of $\mathrm{cBP}$ compared with peripheral $\mathrm{BP}$ in predicting preclinical organ damage. ${ }^{8-10}$

Moreover, evidence from previous studies on multiple populations suggested that central blood pressure is a better predictor of cardiovascular events than brachial pressure. $^{11,12}$

Normally, central BP is lower than brachial values, as a consequence of a physiological phenomenon of amplification, for which an increase in arterial stiffness moving from the highly elastic central arteries of the heart away to the stiffer brachial artery. ${ }^{13}$

Elevated cSBP can, indeed, induce increased heart workload, with consequent left ventricular hypertrophy, decreased perfusion during the diastolic phase, and reduced coronary blood flow. ${ }^{14}$

Previous studies investigated the relationship between salt intake, central BP and arterial stiffness. ${ }^{7,15}$ To date, the gold-standard method to quantify salt intake is the determination of 24-hour urinary sodium excretion. ${ }^{16}$ However, daily variability in the sodium excretion rhythm is a debated field of research. A circadian rhythm of sodium excretion in urine exists and, in physiological conditions, it is characterised by an increased rate of excretion during daytime and a decreased rate during night-time. ${ }^{17}$

Moreover, the daily rate of sodium excretion in the urine showed inter-individual variability, and some evidence suggested the existence of different patterns of day- and night-time sodium excretion. In addition, the daily pattern of sodium excretion could have a different impact on cardiovascular (CV) risk. Higher risk was documented in nondippers and in subjects with increased nighttime BP values showing lower day-time urinary sodium excretion. $^{18,19}$

Recently, it was found that an abnormal circadian rhythm of urinary sodium excretion was independently associated with hypertension and target-organ damage in a selected population affected by chronic kidney disease. ${ }^{20}$

Although a number of studies suggested that daily sodium intake is related to arterial stiffness and central blood pressure, there have been no studies until now exploring whether a difference in the rhythm of sodium excretion could impact arterial-stiffness and central BP components.

In the present study, we sought to elucidate the relationship existing between the day:night urinary sodium excretion ratio, arterial stiffness and central hemodynamics in a general sample of an adult population.

\section{Methods \\ Study Participants}

The present analysis was based on a cross-sectional study of a sample of general-population residents in southern Switzerland (Ticino), conducted between 2017 and 2018. A detailed description of the study design and the main characteristics of the population were published elsewhere. ${ }^{21}$ Briefly, the study aimed to explore vascular ageing and cardiovascular risk factors in the resident population. At the end of the recruitment period, the data of 1202 participants were collected. The local Swiss ethics committee (Comitato etico cantonale Ticino) approved the research protocol, and all participants signed their written consent before data collection. The study protocol adhered to the Declaration of Helsinki. In the present analysis, we excluded 76 individuals on the basis of incomplete urine collection in either the day- or night-time, and 64 individuals for incomplete data from the 24-hour ambulatory blood pressure (ABPM). The present analyses are, therefore, based on 1062 individuals.

\section{Clinical Assessment and Urine Collection}

The study protocol included two consecutive visits with an examination performed during a weekday on the participants' usual diet. Examinations were performed at the research unit of the internal medicine department of the regional hospital of Bellinzona (Switzerland). During the first visit, demographic, comorbidity, traditional cardiovascular risk factors, and medication information were collected. Anthropometric characteristics were assessed: height $(\mathrm{cm})$, weight $(\mathrm{kg})$, waist circumference $(\mathrm{cm})$, hip circumference $(\mathrm{cm})$, and body-mass index (BMI; $\mathrm{kg} / \mathrm{m}^{2}$ ). Peripheral blood pressure (BP) and heart rate $(\mathrm{m} / \mathrm{sec})$ were measured during the first examination using the Mobil-O-Graph and a validated automatic oscillometric 
device (Dinamap model Pro 100 automated sphygmomanometer, Critikon, Tampa, Florida). For consistency, only results of peripheral blood pressure obtained with the MobilO-Graph were shown.

Patients were asked to make the first visit after at least 8 hours of fasting, and the following parameters in serum were determined: blood glucose, total cholesterol, triglyceride, high-density lipoprotein cholesterol, low-density lipoprotein cholesterol, creatinine and cystatin, magnesium, and calcium.

During the first day, tonometric assessment of arterial stiffness was also performed following the procedure described below, and a 24-hour ambulatory-blood-pressure monitoring recording started. At the end of the first examination, participants were given two containers for urine collection, marked as "day-time urine" and "night-time urine", with a beaker to transfer the urine in the collection containers. Day- and night-time periods were defined according to selfreported bed- and wake-up time. A diary was also provided in order to mark (i) the starting diurnal hour of urine collection, (ii) the starting hour of night-time urine collection, and (iii) the hour of the last collected urine sample. All urine voided during the next 24 hours was collected. The next day, the second study/examination day, the collected urine containers were transferred to the Biochemistry Laboratory of Ente Ospedaliero Cantonale, at the regional hospital of Bellinzona, for analyses. In the collected urine, the following parameters were measured: sodium and potassium concentrations, albumin concentration, and creatinine. All urine assessments of day- and night-time periods were separately performed. Self-reported urine loss and 24-hour urine volume were excluded in cases of insufficient urine collection (ie, urine volume $<500 \mathrm{~mL} / 24 \mathrm{~h}$, self-reported loss of a urine sample of more than $100 \mathrm{~mL}$, or more than one time). The amount of excreted 24-hour urine sodium and potassium was calculated by multiplying the total volume of the collected urine by the concentration of measured sodium.

\section{Central Hemodynamics and Arterial Stiffness Assessment}

Estimates of central hemodynamics were noninvasively obtained using a 24-hour ambulatory blood pressure (ABPM) device (Mobil-O-Graph, IEM; Stolberg, Germany) that was able to record pulse waveforms from the brachial artery for 24 hours with an integrated ARC solver algorithm (Austrian Institute of Technology, Vienna). Recorded and analysed (more than $75 \%$ of readings) hemodynamics were central systolic blood pressure (cSBP), central diastolic blood pressure (cDBP), augmentation pressure (AP), augmentation index standardised to a heart rate of $75 \mathrm{bpm}$ (AIx 75), cardiac output $(\mathrm{CO})$, cardiac index $(\mathrm{CI})$, peripheral vascular resistance (PVR), and reflection magnitude percentage (Ref \%). Every study participant underwent 24-hour BP monitoring during the first study visit. Arm circumferences were first measured and recorded to allow for the correct choice of cuff size. The ABPM device consisted of a brachial cuff-based oscillometric monitor, approved and validated for brachial BP monitoring. ${ }^{22}$ The device was programmed to obtain BP recordings every 30 minutes during the day-time and every hour during night-time.

The algorithm used for the oscillometric central BP estimation has already been largely described. Briefly, after standard oscillometric BP assessment, the brachial cuff reinflated, acquiring brachial pressure waveforms for about 10 seconds. Thereafter, a generalised transferfunction algorithm (ARC Solver) produced the aorticpulse waveform after a process of calibration based on brachial SBP and DBP. ${ }^{22}$ Computerised point-and-areabased analysis was further realised to derive different cardiovascular parameters. Analysis of the aortic-pulse waveform was therefore performed, with separate analysis of the incident and reflected waves using an uncalibrated triangular aortic-flow waveform. Pulse-wave velocity was estimated from the time difference between the derived forward and reflected waves. Central hemodynamics parameters provided by the Mobil-O-Graph device were validated in comparative studies with other noninvasive devices (Sphygmocor, ArtCor, Sydney, Australia), as well as with gold-standard invasive intra-aortic techniques. ${ }^{23,24}$ High levels of reproducibility and accuracy were found under both static and ambulatory conditions.

Arterial stiffness was estimated by analysing the goldstandard parameter of pulse-wave velocity (PWV). PWV was noninvasively assessed in each participant with both an oscillometric and a tonometric method. Tonometric cfPWV measurements were performed during the first day of the examination, following a standard operational procedure in a quiet, stable temperature room. After 10 minutes of participants resting in the supine position, as recommended by ARTERY Society guidelines, arterial waveforms at the subject's dominant carotid and femoral sites were recorded by applanation tonometry using a SphygmoCor device (AtCor Medical, Sydney, Australia; Model MM3, Software version 7.01 S). Participants were instructed to abstain from caffeine and tobacco use for four hours before the examination. Measurements with 
a standard deviation of less than $10 \%$ were used for analysis. After cf-PWV measurement, oscillometric pulse-wavevelocity estimation was acquired, and ABPM was applied to each participant.

The oscillometric parameters of PWV were assessed with the ABPM in use, able to simultaneously perform BP and PWV measurements. All PWV and central hemodynamic measurements were performed on a weekday. PWV assessment was first conducted with the tonometric method, and then by the o-PWV device following the described standardised procedures. Considering arterial stiffness as a consequence of a chronic stress of the arterial wall, and the well-documented association between the 24h-ABPM measurements and specific target organ damage (ie left ventricular hypertrophy), ${ }^{25,26}$ the mean 24 hour estimates of PWV and central hemodynamic were used for data analysis.

\section{Statistical Analysis}

Continuous variables were expressed as mean $\pm \mathrm{SD}$, and categorical variables as a percentage. According to the day:night ratio of urinary sodium excretion distribution in the study population, tertile categories were defined as follows: $\mathrm{T} 1<0.85 ; 0.85 \leq \mathrm{T} 2<1.56 \leq \mathrm{T} 3$. Categorical variables were compared using the $\chi^{2}$ test. Variables were compared by tertile group with analysis of variance (ANOVA) for multiple comparisons and Tukey post hoc test or Games-Howell post hoc test were used as appropriate for the comparison of PWVs and hemodynamic parameters among the three groups of day:night ratio of urinary sodium excretion.

The association of day:night ratio of urinary sodium excretion with hemodynamic parameters and arterial stiffness was explored using cSBP, cDBP, cPP, AP, AIx, CI, PVR, Ref \%, o-PWV, and cf-PWV as dependent variables. Both a crude (Model 1) and an adjusted model (Model 2) were provided to examine potential confounding effects. All traditional cardiovascular-risk factors and variables potentially affecting the dependent variables were selected: sex, age, diabetes, hypertension, hypercholesterolemia, current smoking, body-mass index, hip circumference, systolic and diastolic blood pressure, heart rate, cholesterol LDL and HDL, cystatin $\mathrm{C}$, creatinine, magnesium, calcium, pulse pressure, urine potassium; $\beta$-coefficient and confidence interval (CI) are shown. Each adjusted regression model was separately examined for multicollinearity using the variance inflation factor (VIF) statistic; VIF $>4.0$ was an indicator of multicollinearity. To explore the association between the day:night ratio of urinary sodium excretion and the risk of increased arterial stiffness, logistic-regression models were also produced. Associations were expressed as odds ratios (OR) with $95 \%$ confidence interval (CI). Increased arterial stiffness was defined as that with values $\geq 10 \mathrm{~m} / \mathrm{sec}$. Both crude and adjusted models are shown.

Restricted cubic regression spline analyses with three knots located at the 25th, 50th, and 75th percentiles were performed to test the linearity of the relationship between the day:night ratio of urinary sodium excretion and PWV. Values were plotted for values of day:night ratio of urinary sodium excretion until 4 , considering that the low amount of individuals with higher values (n. 13).

All statistical analyses were conducted using SPSS (version 18.0, SPSS Inc, IBM, US) and STATA. P $\leq 0.05$ was considered statistically significant; all P-values were two-tailed.

\section{Results}

Patient characteristics, blood pressure, sodium excretion, and humoral variables for the whole study group and between tertiles of day:night ratio of urinary sodium excretion are presented in Table 1. The subjects in $\mathrm{T} 1$ of sodium excretion could be considered as low day-time sodium excretors, excreting an amount of sodium during the day-time of more than 30\% lower compared with subjects in T3.

However, even if a different intra-daily pattern of urinary sodium excretion was evident, no significant differences in the 24-h urinary sodium excretion were present across tertiles (p-value 0.281).

Mean age \pm SD for the entire sample population was 51.4 \pm 13.6 years, and it significantly increased as day:night ratio of urinary sodium excretion decreased. Subjects in the lowest tertiles of the day:night ratio of urinary sodium excretion also showed significantly higher BMI values (p-value 0.042). Creatinine values reduced and eGFR significantly increased as tertiles decreased ( $p$-value $\leq 0.001$ ). Considering BP parameters, no differences across day:night ratio of urinary sodium excretion for the in-office and daytime BP values for both SBP and DBP were found. Low day-time sodium excretors (T1) showed significantly higher nighttime values of both systolic and diastolic BP, compared with high daytime sodium excretors ( $\mathrm{p}$-value $\leq 0.001$ ). No difference in the rate of hypertension across tertiles was detected.

Comparison of baseline characteristics among the three groups revealed no significant differences in other parameters. 
Table I Participants' Characteristics by Tertiles of Day:Night Ratio of Urinary Sodium Excretion

\begin{tabular}{|c|c|c|c|c|c|}
\hline Variables & Overall & $\begin{array}{l}\text { TI Ratio Day:Night } \\
\text { UrinSodExc }\end{array}$ & $\begin{array}{l}\text { T2 Ratio Day:Night } \\
\text { UrinSodExc }\end{array}$ & $\begin{array}{l}\text { T3 Ratio Day:Night } \\
\text { UrinSodExc }\end{array}$ & p-value \\
\hline Age, years & $51.4 \pm 13.6$ & $54.4 \pm \mid 4.2$ & $51.3 \pm 13.6$ & $48.8 \pm 12.4$ & $\leq 0.001 *$ \\
\hline Females. (\%) & $591(55.7)$ & $182(52)$ & $204(58.3)$ & $205(56.6)$ & 0.221 \\
\hline $\mathrm{BMI}, \mathrm{kg} / \mathrm{m}^{2}$ & $25.0 \pm 4.4$ & $25.4 \pm 4.6$ & $25.2 \pm 4.4$ & $24.6 \pm 4.1$ & $0.042 *$ \\
\hline Current Smoking, (\%) & $192(18.2)$ & $47(13.5)$ & $58(16.7)$ & $86(23.9)$ & $\leq 0.001 *$ \\
\hline Diabetes, (\%) & $22(2.1)$ & $8(2.3)$ & $5(1.5)$ & $9(2.5)$ & 0.582 \\
\hline Hypertension, (\%) & $162(15.4)$ & $63(18.1)$ & $55(15.9)$ & $45(12.5)$ & 0.119 \\
\hline $\mathrm{SBP}, \mathrm{mmHg}$ & $130 \pm 19$ & $|3| \pm 2 \mid$ & $130 \pm 19$ & $129 \pm 18$ & 0.417 \\
\hline $\mathrm{DBP}, \mathrm{mmHg}$ & $85 \pm 13$ & $86 \pm 13$ & $86 \pm 13$ & $85 \pm 13$ & 0.493 \\
\hline Daytime SBP, $\mathrm{mmHg}$ & $122 \pm 12$ & $123 \pm 13$ & $122 \pm 12$ & $121 \pm 12$ & 0.1471 \\
\hline Daytime DBP, mmHg & $77 \pm 9$ & $77 \pm 8$ & $77 \pm 9$ & $77 \pm 9$ & 0.385 \\
\hline Nighttime SBP, mmHg & $112 \pm 13$ & $114 \pm 14$ & $111 \pm 12$ & $109 \pm 11$ & $\leq 0.001 *$ \\
\hline Nighttime DBP, mmHg & $67 \pm 9$ & $69 \pm 10$ & $67 \pm 9$ & $66 \pm 9$ & $\leq 0.001 *$ \\
\hline Heart Rate, beats/min & $69 \pm 11$ & $68 \pm 11$ & $68 \pm 10$ & $69 \pm 10$ & 0.247 \\
\hline Fasting blood Glucose, mmol/L & $5.92 \pm 0.77$ & $5.99 \pm 0.88$ & $5.89 \pm 0.73$ & $5.87 \pm 0.69$ & 0.064 \\
\hline Magnesium, mmol/L & $0.83 \pm 0.06$ & $0.83 \pm 0.06$ & $0.83 \pm 0.06$ & $0.82 \pm 0.05$ & 0.295 \\
\hline Total Cholesterol, mmol/L & $5.35 \pm 1.06$ & $5.28 \pm 1.05$ & $5.37 \pm 1.05$ & $5.39 \pm 1.08$ & 0.305 \\
\hline $\mathrm{HDL}, \mathrm{mmol} / \mathrm{L}$ & $1.57 \pm 0.43$ & $1.59 \pm 0.44$ & $1.57 \pm 0.45$ & $1.57 \pm 0.41$ & 0.741 \\
\hline LDL,mmol/L & $3.60 \pm 1.03$ & $3.53 \pm 1.00$ & $3.61 \pm 1.04$ & $3.66 \pm 1.03$ & 0.223 \\
\hline Triglycerides, $\mathrm{mmol} / \mathrm{L}$ & $1.18 \pm 1.37$ & $1.14 \pm 0.67$ & $1.20 \pm 1.14$ & $1.18 \pm 1.96$ & 0.839 \\
\hline Creatinine, $\mu \mathrm{mol} / \mathrm{L}$ & $75.5 \pm 19.7$ & $79.3 \pm 25.6$ & $75.4 \pm 16.5$ & $71.8 \pm 14.5$ & $\leq 0.001 *$ \\
\hline eGFR,mL/min/l.73m2 & $95.4 \pm 16.9$ & $92.8 \pm 18.1$ & $95.8 \pm 16.6$ & $97.6 \pm 15.6$ & $\leq 0.001 *$ \\
\hline 24 hour $\mathrm{Na}$ excretion, mmol & $138.3 \pm 61.8$ & $142.2 \pm 69.3$ & $139.3 \pm 57.3$ & $134.7 \pm 62.1$ & 0.281 \\
\hline Sodium Excretion daytime, mmol & $73.6 \pm 36.8$ & $57.3 \pm 27.1$ & $74.2 \pm 30.3$ & $88.8 \pm 43.4$ & $\leq 0.001 *$ \\
\hline Sodium Excretion nighttime, mmol & $65.1 \pm 36.4$ & $84.9 \pm 49.7$ & $65.1 \pm 27.5$ & $45.9 \pm 22.8$ & $\leq 0.001 *$ \\
\hline
\end{tabular}

Note: *p-value $<0.05$.

Abbreviations: BMI, body mass index; SBP, systolic blood pressure; DBP, diastolic blood pressure; UrinSodExc, urinary sodium excretion; eGFR, glomerular filtration rate.

Hemodynamic parameters are shown in Table 2. Compared with Group T3, Group T1 displayed higher values of cSBP and cDBP. Low day-time excretors showed significantly higher values of cSBP $(111.6 \pm 12.1)$ compared with high day-time excretors $(110.0 \pm 10.8, \mathrm{p}$-value $\leq 0.001)$, and of cDBP $(76.9 \pm 9.2$ vs $75.1 \pm 9.3 \mathrm{mmHg}$, p-value $\leq 0.001)$. No significant differences were observed between tertiles for other hemodynamic parameters. No remarkable differences were observed between any of the three groups with respect to cPP, AP grades, AIx, cardiac output, cardiac index, peripheral vascular resistance, and reflection magnitude percentage (Ref \%). Comparisons between groups revealed significant differences in cSBP (T1 vs T3) and in cDBP (T1 vs T3). All other comparisons showed no significant differences.

Associations between the day:night ratio of urinary sodium excretion and central hemodynamic parameters were also explored in multivariate-regression models (Table 3). Central BP was significantly associated with the day:night ratio of sodium excretion in both crude (Model 1) and adjusted models (Model 2). Specifically, for the $\operatorname{cSBP}$ ( $\beta$-coefficient, $\mathrm{CI}$ ) of Models 1 and 2, they were, respectively, $-1.655(-2.800,-0.510)$, $\mathrm{p}$-value $\leq 0.001$, and $0.767(-1.520,-0.014) \mathrm{p}$-value 0.046 ; for cDBP, they were $1.319(-2.218,-0.420)$ p-value $\leq 0.001$; and -0.819 ($1.539,-0.099)$, p-value 0.026 . In a similar manner, a significant decrease of cardiac output and peripheral resistances as the day:night ratio of sodium excretion increased was also found in linear-regression models.

Mean \pm SD by tertiles of day:night ratio of sodium excretion (T1 vs T2 vs T3) for cf-PWV were respectively, $7.6 \pm 1.9$ vs $7.3 \pm 1.8$ vs $6.9 \pm 1.5 \mathrm{~m} / \mathrm{sec}, \mathrm{p} \leq 0.001$, as shown in Figure 1A. Low day-time excretors showed significantly higher $\mathrm{T} 1$ values of arterial stiffness, also measured using the oscillometric method (oPWV), which was $7.7 \pm 1.9$ compared with $7.3 \pm 1.7$ and $6.9 \pm 1.5 \mathrm{~m} / \mathrm{sec}$, respectively, in T2 and in T3 (Figure 1B).

Multivariate-regression models confirmed, in both crude and adjusted models, significantly reduced arterial stiffness as day-time urinary sodium excretion increased. More specifically, cf-PWV in Model 1 showed a $\beta$-coefficient (CI) of $-0.386(-0.559,-0.213)$, p-value $\leq 0.001$, with an association that significantly persisted after controlling for multiple confounding factors in Model 2: $0.164(-0.307,-0.022)$, 
Table 2 Comparison of Central BP and Hemodynamic Parameters by Tertiles of Day:Night Ratio of Urinary Sodium Excretion

\begin{tabular}{|c|c|c|c|c|c|}
\hline $\begin{array}{l}\text { Central Aortic Pressure } \\
\text { Parameters }\end{array}$ & $\begin{array}{l}\text { TI Ratio Day:Night } \\
\text { UrinSodExc }\end{array}$ & $\begin{array}{l}\text { T2 Ratio Day:Night } \\
\text { UrinSodExc }\end{array}$ & $\begin{array}{l}\text { T3 Ratio Day: Night } \\
\text { UrinSodExc }\end{array}$ & $F / \chi^{2}$ & p-value \\
\hline cSBP & $112 \pm 12^{*}$ & $110 \pm 11$ & $109 \pm 11^{*}$ & 4.403 & 0.012 \\
\hline CDBP & $77 \pm 9^{¥}$ & $76 \pm 9$ & $75 \pm 9^{¥}$ & 3.823 & 0.022 \\
\hline cPP & $35.7 \pm 12.5$ & $34.6 \pm 11.3$ & $35.3 \pm 11.5$ & 0.880 & 0.415 \\
\hline AP & $10.1 \pm 7.7$ & $9.6 \pm 6.9$ & $9.9 \pm 7.1$ & 0.509 & 0.601 \\
\hline Cardiac Index & $2.5 \pm 0.5$ & $2.6 \pm 0.6$ & $2.6 \pm 0.8$ & 0.602 & 0.548 \\
\hline Cardiac Output & $4.5 \pm 0.51$ & $4.4 \pm 0.48$ & $4.4 \pm 0.49$ & 0.990 & 0.372 \\
\hline Peripheral resistances & $1.30 \pm 0.12$ & $1.29 \pm 0.12$ & $1.29 \pm 0.11$ & 0.919 & 0.399 \\
\hline Refl. Coef & $66.0 \pm 4.5$ & $66.1 \pm 4.0$ & $65.8 \pm 4.8$ & 0.540 & 0.583 \\
\hline Alx(HR75) & $24.6 \pm 7.8$ & $24.7 \pm 8.1$ & $24.0 \pm 8.2$ & 0.720 & 0.487 \\
\hline
\end{tabular}

Notes: ${ }^{*}$-value $<0.05$ according to post hoc testing comparisons $\mathrm{TI}$ vs $\mathrm{T} 3$ and T3 vs TI. Comparisons between groups revealed significant differences in cSBP (TI vs T3) and in CDBP (TI vs T3). All other comparisons were not significant.

Abbreviations: cSBP, central systolic blood pressure; cDBP, central diastolic blood pressure; cPP, central pulse pressure; AP, augmented pressure.

p-value 0.024. A similar significant association was also found for o-PWV.

In logistic regression models, the risk of increased arterial stiffness was significantly reduced as the day:night ratio of urinary sodium excretion increased (Table 4). Specifically, for Models 1 and $2(\mathrm{OR}, 95 \% \mathrm{CI})$, the risk of cf-PWV $\geq 10 \mathrm{~m} /$ sec for the day:night ratio of sodium excretion was, respectively, $0.40(0.25-0.65, \mathrm{p} \leq 0.001)$ and $0.60(0.37-0.98$, p-value 0.039 ). The day:night ratio of sodium excretion was also significantly associated with a reduced risk of increased o-PWV in both logistic models. Restricted cubic spline analysis suggested that the relationship between day:

Table 3 Estimates in Arterial Stiffness Parameters and Central Hemodynamics According to Tertiles of the Urinary Daytime: Nighttime Sodium Excretion Ratio

\begin{tabular}{|c|c|c|c|c|}
\hline \multicolumn{5}{|l|}{ Arterial Stiffness Parameters } \\
\hline & Unadjusted Model & \multirow[t]{2}{*}{ p-value } & Adjusted Model & \multirow[t]{2}{*}{ p-value } \\
\hline & $\begin{array}{l}\beta \text {-Coefficient } \\
\text { (95\%, Confidence Interval) }\end{array}$ & & $\begin{array}{l}\beta \text {-Coefficient } \\
\text { (95\%, Confidence Interval) }\end{array}$ & \\
\hline Cf-PWV & $-0.386(-0.559,-0.213)$ & $\leq 0.00 \mathrm{I} *$ & $-0.164(-0.307,-0.022)$ & $0.024 *$ \\
\hline o-PWV & $-0.50 I(-0.67 I,-0.332)$ & $\leq 0.001 *$ & $-0.045(-0.089,-0.001)$ & $0.044^{*}$ \\
\hline \multicolumn{5}{|c|}{ Central Aortic Pressure Parameters } \\
\hline & Unadjusted model & \multirow[t]{2}{*}{ p-value } & Adjusted Model & \multirow[t]{2}{*}{ p-value } \\
\hline & $\begin{array}{l}\beta \text {-coefficient } \\
(95 \%, \text { confidence interval) }\end{array}$ & & $\begin{array}{l}\beta \text {-coefficient } \\
\text { (95\%, confidence interval) }\end{array}$ & \\
\hline cSBP, mmHg & $-1.655(-2.800,-0.510)$ & $\leq 0.001 *$ & $-0.767(-1.520,-0.014)$ & $0.046^{*}$ \\
\hline cDBP, $\mathrm{mmHg}$ & $-1.319(-2.218,-0.420)$ & $\leq 0.001 *$ & $-0.819(-1.539,-0.099)$ & $0.026^{*}$ \\
\hline cPP, mmHg & $0.577(-0.654, I .808)$ & 0.358 & $0.217(-0.915-1.349)$ & 0.707 \\
\hline $\mathrm{AP}, \mathrm{mmHg}$ & $0.136(-0.610-0.882)$ & 0.676 & $0.312(-0.422-1.046)$ & 0.404 \\
\hline Cardiac Index, $1 / \mathrm{min}^{*} \mathrm{I} / \mathrm{m}^{2}$ & $-0.025(-0.03 \mathrm{I}-0.08 \mathrm{I})$ & 0.378 & $-0.029(-0.03-0.026)$ & 0.303 \\
\hline Cardiac Output, I/min & $-0.054(-0.105,-0.004)$ & $0.034 *$ & $-0.070(-0.109,-0.032)$ & $\leq 0.00 I^{*}$ \\
\hline Peripheral resistances, $\mathrm{mmHg} / \mathrm{mL}$ & $-0.002(-0.014-0.010)$ & 0.779 & $0.012(0.001-0.023)$ & $0.033^{*}$ \\
\hline Refl. Coef, \% & $-0.231(-0.705-0.243)$ & 0.339 & $0.012(-0.416-0.439)$ & 0.957 \\
\hline Alx(HR75), \% & $-0.442(-1.252-0.367)$ & 0.284 & $0.162(-0.390-0.713)$ & 0.565 \\
\hline
\end{tabular}

Notes: Model I unadjusted; Model 2 adjusted for sex, age, diabetes, hypertension, hypercholesterolemia, current smoking, body mass index, hip circumference, systolic blood pressure, diastolic blood pressure, heart rate, cholesterol LDL, cholesterol HDL, triglycerides, cystatin C, creatinine, magnesium, calcium, pulse pressure, fasting plasma glucose. ${ }^{*} \mathrm{p}$ value $<0.05$.

Abbreviations: cf-PWV, carotid-femoral pulse wave velocity; o-PWV, oscillometric pulse wave velocity; cSBP, central systolic blood pressure, cDBP, central diastolic blood pressure, cPP central pulse pressure, AP, augmented pressure, Alx(HR75), augmentation index corrected for heart rate 75 beat per minute. 
night ratio and PWV prolongation was not linear (nonlinearity $p<0.001$ ) (Figure 2).

\section{Discussion}

The novel finding of the present study is that the pattern of urinary sodium excretion, characterised by low day-time excretion, is associated with increased arterial stiffness and augmented central hemodynamic parameters such as cSBP, cDBP, and cardiac output in the general population. In addition, the risk of documented vascular target-organ damage significantly decreased as day-time sodium excretion increased. These results provide observational evidence that the intraindividual pattern of urinary sodium excretion is associated with central blood pressure and arterial ageing.

The close relationship between high sodium intake with diet and cardiovascular health has been well explored, and several previous studies have highlighted its negative effects on cardiovascular outcomes. ${ }^{27,28}$

However, it is also important to identify factors that could affect CVD risk at the individual level, considering that CVD is still globally one of the most frequent causes of death. From this perspective, a better understanding of renal sodium-handling mechanisms could contribute to providing prevention measures with individualised programmes of salt intake that also could take into account the subject's pattern of intradaily urinary sodium excretion.

Excessive sodium intake negatively impacts blood pressure, and significantly increases the risk of stroke and renal dysfunction. ${ }^{29,30}$ Moreover, findings from both

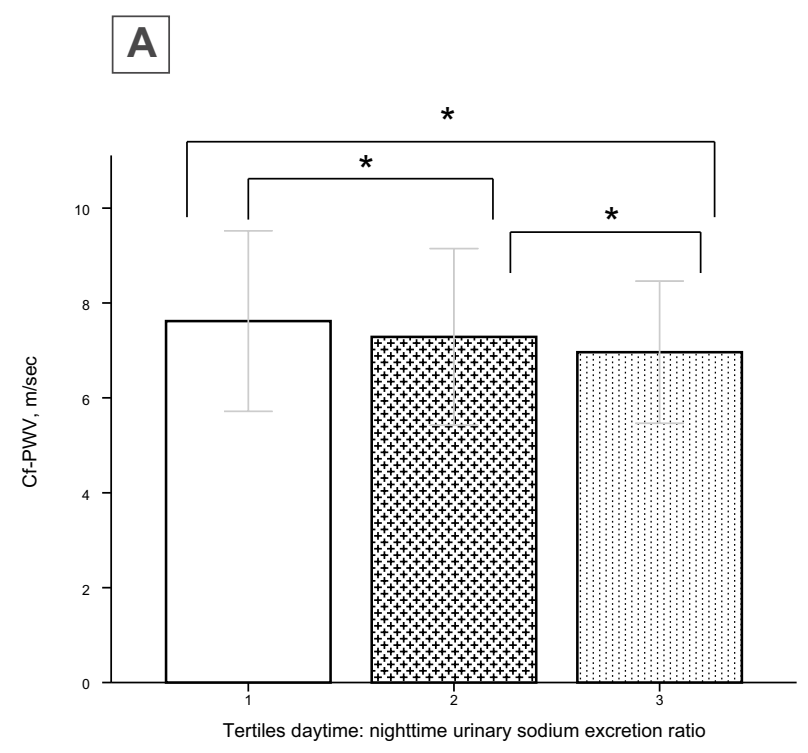

epidemiological and clinical studies indicated an association between habitual dietary-salt intake and PWV. ${ }^{31,32}$

The salt-mediated BP increase is a multifactorial phenomenon in which complex inter-related mechanisms are implied, ranging from the action of sodium on small-artery resistance to a direct effect on the structure and function of large arteries. $^{33}$ Considering the above-mentioned saltinduced mechanisms, increased arterial stiffness represents a BP-independent consequence of mean increased salt intake which also partially contributes to BP increase.

However, BP and PWV are two factors that are strictly interdependent, and a robust number of cardiovascularphysiology studies have highlighted this association in which complex and inter-related mechanisms are involved. This hypothesis is also corroborated by recent metaanalysis of available randomised controlled trials in which direct association between the reduction of dietarysalt intake and a decrease in arterial stiffness was found. ${ }^{32}$ It was highlighted that an average weighed difference of $89.3 \mathrm{mmol}$ of sodium per day corresponded, in the short term (1-6 weeks), to a $2.8 \%$ reduction in arterial stiffness. It was also shown that PWV reduction was independent from BP reduction. ${ }^{15}$ Furthermore, experimental evidence confirmed also the BP-independent nature of salt-induced vascular alterations. ${ }^{34}$ It was identified that vascular damage consequent to salt intake could be linked to an abnormal activation of the renin-angiotensin system, and with reduced bioavailability of nitric oxide. ${ }^{35}$ Even if the relationship between salt consumption and arterial

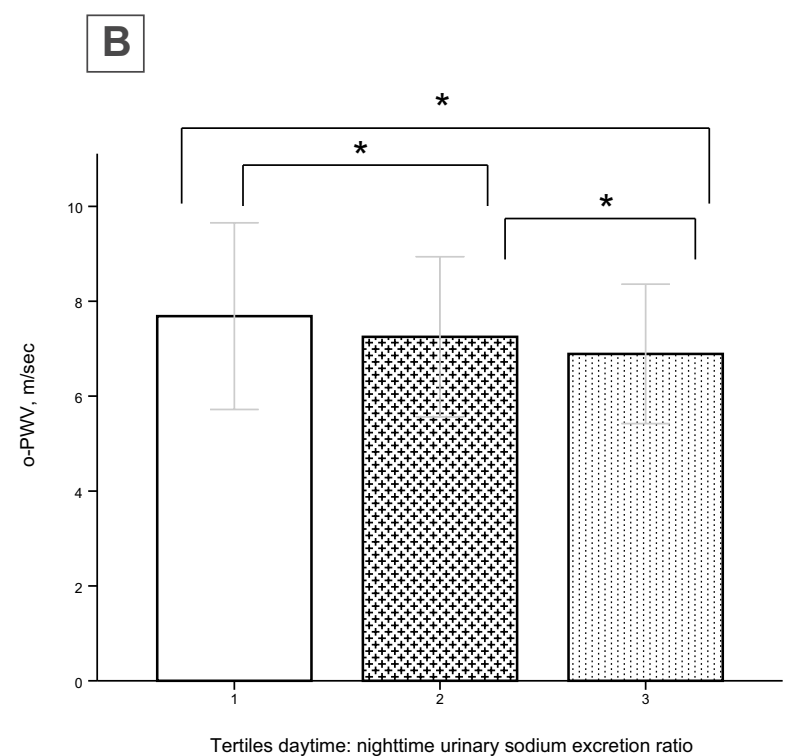

Figure I Comparison of oscillometric and carotid-femoral pulse wave velocity (o-PWV and cf-PWV) among the tertiles of day:night ratio of urinary sodium excretion. CfPWV (A) and o-PWV (B) means \pm SD by tertiles of daytime: nighttime urinary sodium excretion ratio. *Indicates p-value $<0.05$ according to post hoc testing comparison. 
Table 4 Logistic Regression Models Exploring the Risk of Increased Arterial Stiffness and Day:Night Ratio of Urinary Sodium Excretion

\begin{tabular}{|c|c|c|c|}
\hline & \multicolumn{3}{|c|}{ Risk of Increased cf-PWV } \\
\hline & OR & $95 \% \mathrm{Cl}$ & p-value \\
\hline \multirow{3}{*}{ Day:night ratio of urinary sodium excretion } & \multicolumn{3}{|c|}{ Model I } \\
\hline & 0.40 & $0.25-0.65$ & $\leq 0.001$ \\
\hline & Mode & & \\
\hline \multirow[t]{2}{*}{ Day:night ratio of urinary sodium excretion } & 0.60 & $0.37-0.98$ & 0.041 \\
\hline & \multicolumn{3}{|c|}{ Risk of increased o-PWV } \\
\hline \multirow{3}{*}{ Day:night ratio of urinary sodium excretion } & \multicolumn{3}{|c|}{ Model I } \\
\hline & 0.25 & $0.14-0.43$ & $\leq 0.001$ \\
\hline & \multicolumn{3}{|c|}{ Model 2} \\
\hline Day:night ratio of urinary sodium excretion & 0.27 & $0.072-0.99$ & 0.048 \\
\hline
\end{tabular}

Abbreviations: cf-PWV, carotid-femoral pulse wave velocity; o-PWV, oscillometric pulse wave velocity. Model I, unadjusted; Model 2, adjusted for sex, age, previous cardiovascular disease, diabetes, hypertension, hypercholesterolemia, current smoking, body mass index, hip circumference, systolic blood pressure, diastolic blood pressure, heart rate, cholesterol LDL, cholesterol HDL, triglycerides, cystatin C, creatinine.

stiffness has been previously investigated, no studies examined the association between the daily pattern of urinary sodium excretion and arterial stiffness.

As previous studies showed, the low day-time pattern of sodium excretion is significantly associated with increased $\mathrm{CV}$ risk by affecting the nondipping pattern in hypertensive and nonhypertensive subjects. ${ }^{17}$ Here, we strove to corroborate the compelling hypothesis that the individual pattern of intradaily sodium excretion could affect $\mathrm{CV}$ risk, influencing arterial ageing and central BP parameters.

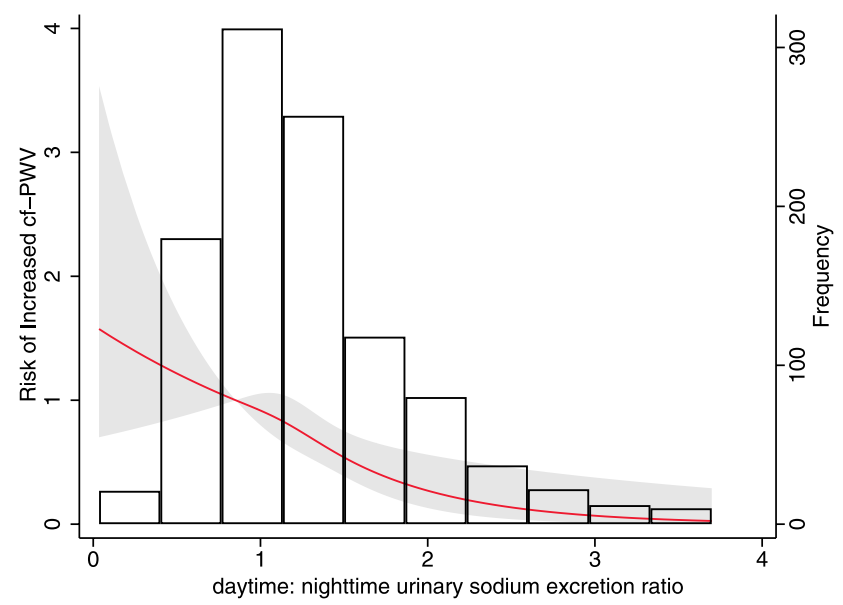

Figure 2 Association between the risk of increased arterial stiffness and the day: night ratio of urinary sodium excretion based on restricted cubic spline model. The bar plots show the day:night ratio of urinary sodium excretion distribution in the population.
In our study, the association between arterial stiffness, measured with PWV, and sodium excretion was significant using two different assessment techniques, aspect which further strengthen the association found.

Moreover, the absence of a significant difference in the 24-h urinary sodium excretion across tertiles, confirms the pivotal role-played by the circadian rhythm of sodium excretion in vascular damage.

As in previous studies, our analysis found no association between augmentation index and sodium excretion. As in previous studies, our analysis found no association between augmentation index and sodium excretion. This aspect could, in part, be explained by the fact that augmentation index strongly depends on many factors beyond vascular-wall properties (ie, systolic ejection duration, $\mathrm{HR}$, and vasoconstriction of peripheral arteries), which can vary without any alteration of aortic stiffness. The reliability of AI as a specific marker of arterial stiffness is still debated. ${ }^{36-38}$ On the other hand, central pressure is significantly associated with the day:night ratio of urinary sodium excretion, with values significantly decreasing as day-time sodium excretion increases. Very few previous studies investigated the association between central BP and hemodynamic parameters, and sodium excretion, and none explored the relationship between the daily pattern of sodium excretion and central BP parameters; even if cBP is more relevant than peripheral BP in CVD pathogenesis. Here, we found that the peculiar pattern of 
low day-time sodium excretors was negatively associated with central BP. Moreover, it is important to note that the association between central pressure values and the daytime pattern of sodium excretion persisted also after correction for peripheral BP measurements in the multivariate analysis. However, due to the observational nature of the present study, we could not identify the exact mechanisms underlying this association. It was previously postulated that sodium loading is associated with an increased level of reactive oxygen species and with a reduced bioavailability of nitric oxide, which is a regulator of the arterial diameter. The impairment of nitric oxide releasing could result in increased wave reflection; it was also shown that sodium loading could affect artery and microvascular function. $^{38}$

The present study should be interpreted within the context of its limitations. The first limitation, as stated above, is the observational cross-sectional nature of the design; not allowing us to establish the cause-effect relationship between the rhythm of urinary sodium excretion, arterial stiffness, central BP, and hemodynamic parameters, and to answer the causality dilemma. A large number of subjects in our study as well as controlling for several potential confounders in multivariate-regression analyses improved result reliability. Lastly, difference in the measured and calculated parameters between the groups was quantitatively small, raising the question of clinical relevance. Moreover, we did not evaluate the longterm influence of sodium intake on central BP and arterial stiffness.

The urine collection was performed on a single day for practical reasons of feasibility. However, even if the 24hour urine sodium excretion is more precise in assessing salt intake, compared with questionnaires and urine overnight samples, we did not perform repeated collections or verify results with questionnaires and therefore, we cannot quantify the inter-daily variability, which could have impacted the results.

The duration of urine collection was not standardised for day- and night-time-period collection, but adapted to intraindividual subject habits, and a variation in collection hours might have also affected the results.

Moreover, an increasing body of studies has highlighted that low sodium and high potassium intake exerts a synergistic effect on BP and related aspects, and that this could be more relevant than the effects of sodium or potassium taken individually. ${ }^{39}$ In the present study, we primarily aimed to investigate the association between the intra-daily variation of sodium excretion, arterial stiffness and central hemodynamics; however, even if beyond our scope, a role of potassium excretion in the association found can be postulated.

Last but not least, the central BP determined with the oscillometric device used in the present study, was based on brachial waveforms calibrated with SBP and DBP. This aspect could limit the reliability of our results, considering the amount of evidence which has currently shown that calibration with mean arterial pressure and DBP provides a more accurate assessment of central $\mathrm{BP}^{40}$

In conclusion, the present findings shed light on an important aspect of the complex relationship between salt intake, arterial ageing, central BP and hemodynamics: not only could the level of sodium intake have significant impact on this relationship but also individual intradaily sodium handling, determining different risk profiles. Possibly the individual daily pattern of sodium excretion should not be disregarded when cardiovascular assessment is performed. However, for deeper insight, further mechanistic and epidemiological investigations in other populations, as well as randomised controlled trials, are advocated to clarify the utility of assessing the daily pattern of sodium excretion in modelling the individual's $\mathrm{CV}$ risk.

\section{Abbreviations}

PWV, pulse wave velocity; BP, blood pressure; cSBP, central systolic blood pressure; cDBP, central diastolic blood pressure; CV, cardiovascular; ABPM, 24-hour ambulatory blood pressure monitoring; BMI, body mass index; AP, augmentation pressure; AIx75, augmentation index standardised to heart rate of $75 \mathrm{bpm}$; $\mathrm{CO}$, cardiac output; $\mathrm{CI}$, cardiac index; PVR, peripheral vascular resistance; Ref \%, reflection magnitude percentage; OR, odds ratios; SD, standard deviation.

\section{Ethics Approval}

The study was carried out in accordance with the Helsinki Declaration and was approved by the local Swiss ethics committee (Comitato etico cantonale Ticino). All participants provided informed written consent.

\section{Acknowledgments}

We would like to thank the participants in the TEST study for their valuable and active contribution and Dr. Giorgio Merlani of the Department of Public Health of the Ticino canton, for his valuable support. We would like also to thank the study nurse Natasa Bettosini for her support in the data collection, Ms. Irene Menghini e her staff for data entry. Salvatore 
Assenzio for the graphic art assistance. We would like to thank Mrs Garo Marialuisa for helpful statistical management.

\section{Author Contributions}

All authors substantially contributed to the conception, design, acquisition of data, analysis and interpretation of data; took part in drafting the article or revising it critically for important intellectual content; gave final approval of the version to be published; and agree to be accountable for all aspects of the work.

\section{Funding}

We gratefully acknowledge for the financial support of the Carlo Gianella Foundation for Clinical Research (Locarno, Switzerland). The funding source had no role in study design, data collection, data analysis, data interpretation, or writing of the report.

\section{Disclosure}

The authors declare no conflicts of interest for this work.

\section{References}

1. O'Donnell M, Mente A, Yusuf S. Sodium intake and cardiovascular health. Circ Res. 2015;116(6):1046-1057. doi:10.1161/ CIRCRESAHA.116.303771

2. Zeng ZH, Luo BH, Gao YJ, et al. Control of vascular changes by renin-angiotensin-aldosterone system in salt-sensitive hypertension. Eur J Pharmacol. 2004;503(1-3):129-133. doi:10.1016/j.ejphar. 2004.09.022

3. Sanders PW. Vascular consequences of dietary salt intake. Am J Physiol Renal Physiol. 2009;297:F237-43. doi:10.1152/ajprenal. 00027.2009

4. Boegehold MA. The effect of high salt intake on endothelial function: reduced vascular nitric oxide in the absence of hypertension. $J$ Vasc Res. 2013;50(6):458-467. doi:10.1159/000355270

5. Ying W-Z, Sanders PW. Dietary salt increases endothelial nitric oxide synthase and TGF-b1 in rat aortic endothelium. Am $J$ Physiol. 1999;277(4):H1293-H1298. doi:10.1152/ajpheart.1999.277.4.H1293

6. Ying W-Z, Sanders PW. Dietary salt modulates renal production of transforming growth factor-b in rats. Am J Physiol. 1998;274(4): F635-F641. doi:10.1152/ajprenal.1998.274.4.F635

7. Muth BJ, Brian MS, Chirinos JA, Lennon SL, Farquhar WB, Edwards DG. Central systolic blood pressure and aortic stiffness response to dietary sodium in young and middle-aged adults. $J \mathrm{Am}$ Soc Hypertens. 2017;11(10):627-634. doi:10.1016/j.jash.2017.07.010

8. Avolio A. Central aortic blood pressure and cardiovascular risk: a paradigm shift? Hypertension. 2008;51(6):1470-1471. doi:10.1 161/HYPERTENSIONAHA.107.108910

9. Vlachopoulos C, Aznaouridis K, O’Rourke MF, Safar ME, Baou K, Stefanadis C. Prediction of cardiovascular events and all-cause mortality with central haemodynamics: a systematic review and meta-analysis. Eur Heart J. 2010;31:1865-1871. doi:10.1093/eurheartj/ehq024

10. Kollias A, Lagou S, Zeniodi ME, Boubouchairopoulou N, Stergiou GS. Association of central versus brachial blood pressure with target-organ damage: systematic review and meta-analysis. Hypertension. 2016;67(1):183-190. doi:10.1161/HYPERTENSION AHA.115.06066
11. Zhang L, Wang Z, Chen Z, et al. Central aortic systolic blood pressure exhibits advantages over brachial blood pressure measurements in chronic kidney disease risk prediction in women. Kidney Blood Press Res. 2018;43(4):1375-1387. doi:10.1159/000492952

12. Roman MJ, Devereux RB, Kizer JR, et al. Central pressure more strongly relates to vascular disease and outcome than does brachial pressure: the strong heart study. Hypertension. 2007;50(1):197-203. doi:10.1161/HYPERTENSIONAHA.107.089078

13. McEniery CM, Cockcroft JR, Roman MJ, Stanley S, Franklin SS, Wilkinson IB. Central blood pressure: current evidence and clinical importance. Eur Heart J. 2014;35(26):1719-1725. doi:10.1093/eurheartj/eht565

14. Roman MJ, Devereux RB. Association of central and peripheral blood pressures with intermediate cardiovascular phenotypes. Hypertension. 2014;63(6):1148-1153. doi:10.1161/HYPERTENSIONAHA.114.03361

15. D’Elia L, Galletti F, La Fata E, Sabino P, Strazzullo P. Effect of dietary sodium restriction on arterial stiffness: systematic review and meta-analysis of the randomized controlled trials. J Hypertens. 2018;36(4):734-743. doi:10.1097/HJH.0000000000001604

16. Wielgosz A, Robinson C, Mao Y, et al. The impact of using different methods to assess completeness of 24-hour urine collection on estimating dietary sodium. J Clin Hypertens (Greenwich). 2016;18 (6):581-584. doi:10.1111/jch.12716

17. Firsov D, Bonny O. Circadian regulation of renal function. Kidney Int. 2010;78(7):640-645. doi:10.1038/ki.2010.227

18. Bankir L, Bochud M, Maillard M, Bovet P, Gabriel A, Burnier M. Nighttime blood pressure and nocturnal dipping are associated with daytime urinary sodium excretion in African subjects. Hypertension. 2008;51(4):891-898. doi:10.1161/HYPERTENSIONAHA.107.1055 10

19. Birkenhäger AM, van den Meiracker AH. Causes and consequences of a non-dipping blood pressure profile. Neth J Med. 2007;65 (4):127-131.

20. Zhang J, Rao J, Liu M, et al. Abnormal circadian rhythm of urinary sodium excretion correlates closely with hypertension and target organ damage in Chinese patients with CKD. Int $J$ Med Sci. 2020;17(6):702-711. doi:10.7150/ijms.42875

21. Del Giorno R, Gabutti S, Troiani C, et al. Association between HDL cholesterol and QTc interval: a population-based epidemiological study. J Clin Med. 2019;8(10):1527. doi:10.3390/jcm8101527

22. Wei W, Tölle M, Zidek W, van der Giet M. Validation of the mobil-O-Graph: 24 h-blood pressure measurement device. Blood Press Monit. 2010;15(4):225-228. doi:10.1097/MBP.0b013e328338892f

23. Wassertheurer S, Kropf J, Weber T, et al. A new oscillometric method for pulse wave analysis: comparison with a common tonometric method. J Hum Hypertens. 2010;24(8):498-504. doi:10.1038/ jhh. 2010.27

24. Hametner B, Wassertheurer S, Kropf J, Mayer C, Eber B, Weber T. Oscillometric estimation of aortic pulse wave velocity: comparison with intra-aortic catheter measurements. Blood Press Monit. 2013;18:173-176. doi:10.1097/MBP.0b013e3283614168

25. Protogerou AD, Argyris AA, Papaioannou TG, et al. Left-ventricular hypertrophy is associated better with 24-h aortic pressure than 24-h brachial pressure in hypertensive patients: the SAFAR study. $J$ Hypertens. 2014;32:1805-1814. doi:10.1097/HJH.000000000000 0263

26. Weber T, Wassertheurer S, Schmidt-Trucksäss A, et al. Relationship between 24-hour ambulatory central systolic blood pressure and left ventricular mass: a prospective multicenter study. Hypertension. 2017;70(6):1157-1164. doi:10.1161/HYPERTENSIONAHA.117.09 917

27. Zhu Y, Zhang J, Li Z, et al. Association of sodium intake and major cardiovascular outcomes: a dose-response meta-analysis of prospective cohort studies. BMC Cardiovasc Disord. 2018;18(1):192. doi:10.1186/s12872-018-0927-9 
28. Cappuccio FP, Beer M, Strazzullo P; European Salt Action Network. Population dietary salt reduction and the risk of cardiovascular disease. A scientific statement from the European Salt Action Network. Nutr Metab Cardiovasc Dis. 2018;29(2):107-114. doi:10.1016/j. numecd.2018.11.010

29. Li XY, Cai XL, Bian PD, Hu LR. High salt intake and stroke: meta-analysis of the epidemiologic evidence. CNS Neurosci Ther 2012;18(8):691-701. doi:10.1111/j.1755-5949.2012.00355.x

30. Garofalo C, Borrelli S, Provenzano M, et al. Dietary salt restriction in chronic kidney disease: a meta-analysis of randomized clinical trials. Nutrients. 2018;10(6):pii: E732. doi:10.3390/nu10060732

31. Pase MP, Grima NA, Sarris J. The effects of dietary and nutrient interventions on arterial stiffness: a systematic review. Am J Clin Nutr. 2011;93:446-454. doi:10.3945/ajcn.110.002725

32. Safar ME, Temmar M, Kakou A, Lacolley P, Thornton SN. Sodium intake and vascular stiffness in hypertension. Hypertension. 2009;54:203-209.17. doi:10.1161/HYPERTENSIONAHA.109.129585

33. Edwards DG, Farquhar WB. Vascular effects of dietary salt. Curr Opin Nephrol Hypertens. 2015;24(1):8-13. doi:10.1097/MNH.000 0000000000089

34. Simon G. Experimental evidence for blood pressure-independent vascular effects of high sodium diet. Am J Hypertens. 2003;16 (12):1074-1078. doi:10.1016/j.amjhyper.2003.07.019

35. Drenjančević-Perić I, Jelaković B, Lombard JH, Kunert MP, Kibel A, Gros M. High-salt diet and hypertension: focus on the renin-angiotensin system. Kidney Blood Press Res. 2011;34 (1):1-11. doi: $10.1159 / 000320387$
36. Lacy PS, O'Brien DG, Stanley AG, Dewar MM, Swales PP, Williams B. Increased pulse wave velocity is not associated with elevated augmentation index in patients with diabetes. J Hypertens. 2004;22:1937-1944. doi:10.1097/00004872-200410000-00016

37. Lemogoum D, Flores G, Van den AW, et al. Validity of pulse pressure and augmentation index as surrogate measures of arterial stiffness during beta-adrenergic stimulation. J Hypertens. 2004;22:511-517. doi:10.1097/00004872-200403000-00013

38. Greaney JL, DuPont JJ, Lennon-Edwards SL, Sanders PW, Edwards DG, Farquhar WB. Dietary sodium loading impairs microvascular function independent of blood pressure in humans: role of oxidative stress. J Physiol. 2012;590(Pt 21):5519-5528. doi:10.1113/ jphysiol.2012.236992

39. Perez V, Chang ET. Sodium-to-potassium ratio and blood pressure, hypertension, and related factors. Adv Nutr. 2014;5(6):712-741. doi:10.3945/an.114.006783

40. Papaioannou TG, Karageorgopoulou TD, Sergentanis TN, et al. Accuracy of commercial devices and methods for noninvasive estimation of aortic systolic blood pressure a systematic review and meta-analysis of invasive validation studies. $J$ Hypertens. 2016;34:1237-1248. doi:10.1097/HJH.0000000000000921

\section{Publish your work in this journal}

Diabetes, Metabolic Syndrome and Obesity: Targets and Therapy is an international, peer-reviewed open-access journal committed to the rapid publication of the latest laboratory and clinical findings in the fields of diabetes, metabolic syndrome and obesity research. Original research, review, case reports, hypothesis formation, expert opinion and commentaries are all considered for publication. The manuscript management system is completely online and includes a very quick and fair peer-review system, which is all easy to use. Visit http://www.dovepress.com/testimonials.php to read real quotes from published authors. 